\title{
Aproximación al uso del halago en el español de Monterrey
}

\author{
INTRODUCTION TO THE USE OF COMPLIMENTS IN MONTERREY SPANISH \\ Elena Jiménez Martin y Eva Mendieta Garrote \\ Universidad Tecnológica de Monterrey \\ Indiana University Northwest \\ Resumen:
}

Este trabajo pretende ser una contribución al conocimiento del uso del halago en la comunidad mexicana de Monterrey. Para ello, se ha llevado a cabo el análisis pragmático de dos corpus lingüísticos distintos y complementarios, compuestos de entrevistas semiformales y de muestras de habla coloquial espontánea. El estudio de la tipología funcional de los halagos recogidos, junto a los tipos de respuesta que suscitan, arroja luz sobre el valor sociocultural de estos actos de habla. En el sistema de cortesía mexicano, los cumplidos se erigen como actos valorizantes que realzan la imagen del otro y tienen al mismo tiempo una repercusión positiva en la imagen propia, con lo que sirven para reforzar la imagen de filiación y acercamiento que caracteriza a la cultura mexicana. Se conforman, pues, como un elemento importante de interacción social, que tiene su base en la cortesía, apego al grupo, a la comunidad y a la comunión con el otro.

PALABRAS CLAVE: (des)cortesía verbal, español de México, el halago, pragmática sociocultural

\section{Abstract:}

The purpose of this paper is to contribute to the understanding of the use of compliments in the Mexican community of Monterrey. To this end, we have analyzed two distinct and complementary linguistic corpora, comprised of semi-formal interviews and spontaneous conversational speech. The functional typology of the compliments identified, along with the study of the responses they receive, both shed light on the sociocultural value of these speech acts. In the Mexican politeness system, compliments 
stand as valorizing actions that enhance the image of the other and simultaneously have a positive impact on self-image, thus serving to reinforce the image of solidarity and closeness that characterizes Mexican culture. In this fashion, compliments constitute an important element of social interaction, which is based on politeness, attachment to the group, and communion with the other.

KEYWORDS:Verbal Politeness, Mexican Spanish, Compliments, Sociocultural Pragmatics

\section{INTRODUCCIÓN}

Las investigaciones sobre el cumplido y la respuesta al cumplido en México son todavía escasas, si bien en los últimos años han aparecido varios estudios que comienzan a sentar las bases para la comprensión del funcionamiento de estos actos de habla en la comunidad mexicana (Reyes 2014; Rodríguez y Jiménez 2010, 2014; Flores 2014; Félix- Brasdefer 2009). El presente trabajo pretende ser una aportación a este conocimiento y se dedica al estudio del cumplido en la ciudad norteña de Monterrey. Para ello, se ha llevado a cabo el análisis de dos corpus lingüísticos distintos y complementarios, compuestos de entrevistas semiformales y de muestras de habla coloquial espontánea. Creemos que el estudio de la tipología funcional de los halagos recogidos, junto a los tipos de respuesta que suscitan, arrojará luz sobre el valor sociocultural de estos actos de habla.

Nuestro estudio se sustenta en los presupuestos básicos que resaltan la importancia del contexto socio-cultural en la producción e interpretación de los enunciados, a la vez que se rechaza el modelo universalista (Bravo, 2010: 119). Bravo (2005: 33-34) define la cortesía como una estrategia que se usa para quedar bien con el otro y que se interpreta como cortés cuando sigue los códigos y las normas sociales establecidos por una comunidad de habla. Al estar dictadas estas normas por los patrones sociales de cada comunidad, varían de cultura en cultura tanto en su manifestación formal como en su función interactiva (Haverkate 1994). Necesitamos, por tanto, partir del reconocimiento de los valores culturales específicos. Para el contexto mexicano, contamos ya con los importantes resultados de estudios recientes sobre cortesía y hábitos sociales (Félix- Brasdefer 2009; Hernández 2004). En particular, la literatura reciente hace uso de las nociones de respeto, confianza y reciprocidad para explicar elecciones discursivas motivadas por cuestiones de cortesía en México. En este trabajo nos concentramos en el estudio del cumplido, entendido como todo acto de habla mediante el que un hablante "emite un enunciado que valora positivamente los méritos o propiedades de otra persona" (Bustos, 2007: 190). En términos de imagen social, se trata de actos de habla "que se orientan hacia el trabajo de la imagen positiva del oyente" (Curcó 2014:34). En este estudio presentamos una clasificación de los ejemplos de halago aparecidos en dos corpus de habla real y proponemos una interpretación de su significado como elemento estratégico en las relaciones sociales entre los hablantes mexicanos, más específicamente en el contexto de la comunidad de Monterrey.

\section{DESCRIPCIÓN DEL CORPUS}


El estudio incluye materiales procedentes de dos corpus bien diferenciados:

1) Corpus del habla de Monterrey-PRESEEA. Se trata de 24 entrevistas semiformales a informantes de edades comprendidas entre los 18 y 35 años, con una duración media de una hora cada entrevista; 2) Corpus AMERESCO. Incluye 24 grabaciones de conversaciones espontáneas entre hablantes de 19 a 30 años de edad. La duración aproximada de cada grabación es de 30 minutos. La incorporación del corpus AMERESCO nos permite analizar por primera vez un corpus de habla espontánea y coloquial real y contrarresta así las limitaciones metodológicas que se asocian con la aparición espontánea de cumplidos en el contexto de las entrevistas de PREESEA, en las que, debido a la temática, el contexto y la relación entre entrevistador/entrevistado, las apariciones del halago son más escasas y cortas, alejadas de la espontaneidad de los intercambios interactivos genuinos de las conversaciones cotidianas.

\section{ALGUNOS PRESUPUESTOS TEÓRICOS}

La teoría de la imagen social o face, con sus conceptos de imagen y actividad de imagen (facework) marca el comienzo de los estudios sobre (des)cortesía. De acuerdo con la noción de imagen de Goffman, el comportamiento verbal de una persona funciona como índice de "su punto de visa de la situación y a través de ésta su evaluación de los participantes, y en particular de sí misma" (Goffman 1967: 21). Por lo tanto, cuando entramos en negociación con otras personas en situaciones específicas, "desarrollamos actividades de imagen mediante las cuales negociamos nuestras intenciones con el interlocutor" (Félix-Brasdefer 2009: 12). Brown y Levinson (1985) propusieron el carácter universal del concepto de imagen social y abrieron el camino a una investigación en la que la manifestación de la imagen social y las actividades de imagen pasan a ocupar un lugar central en el estudio de la cortesía verbal. Cuando emite un enunciado, el hablante no sólo transmite un contenido, sino que, al hacerlo, necesariamente negocia el valor de su propia imagen y la de su interlocutor. La imagen pública (face) se articula en la tensión entre dos necesidades simultáneas: la necesidad de distancia de los otros, de disponer de suficiente "espacio" personal (imagen negativa) y la necesidad de comunión con los otros, de que nos aprecien y aprueben (imagen positiva). "El supuesto fundamental a partir del cual se han construido las teorías sobre la cortesía verbal (...) es que para mantener el equilibrio de una comunidad es indispensable que en cada contacto interpersonal se manifieste de algún modo que estas dos necesidades universales están siendo atendidas por los participantes" (Curcó 2014:40). Estudios posteriores han puesto de manifiesto que las necesidades de imagen de los hablantes varían según la cultura, y distan mucho de ser universales. Fant (1989) y Bravo (1999) propusieron sustituir la imagen positiva y negativa de Brown y Levinson por los conceptos generales de autonomía y afiliación, en los que hallan cabida situaciones culturales no contempladas en la dicotomía anterior. Bravo (1999: 114) aboga por el uso de "unos conceptos vacíos, más generales y relativos, que cada cultura pueda matizar según su propia realidad sociocultural", de forma que cada cultura otorgará diferentes contenidos a cada componente de la imagen. Entre los dos polos que constituyen la defensa de la autonomía y el deseo de afiliación con el otro, existe un continuum 
que cada cultura negocia de manera específica. Félix-Brasdefer (2009), por su parte, utiliza las dos dimensiones de la imagen social desarrolladas por Scollon y Scollon (2001): la imagen de afiliación (involvement) y la imagen de independencia (independence). La manifestación de una u otra imagen dependerá de lo que asuman en cada situación los interlocutores, así como de la distancia social y del poder que exista entre ellos. La imagen de afiliación "se manifiesta al apoyar el punto de vista del interlocutor, mostrar interés en la otra persona, ser parte del grupo y expresar reciprocidad con el interlocutor" (Félix-Brasdefer 2009: 22) y se plasma, por ejemplo, con el uso de formas de tratamiento informales, de un dialecto coloquial, etc., formas que expresan conexión y confianza con el interlocutor. Por el contrario, la imagen de independencia "se expresa al proyectar la individualidad del hablante, recibir y expresar respeto hacia los demás y proyectar sus derechos de autonomía que lo diferencian de los demás" (Félix-Brasdefer 2009: 23); ésta se expresaría por ejemplo con el uso de formas impersonales o de la forma "usted". De cualquier forma, la afiliación y la independencia se presentan en gradación y no deben entenderse en sentido absoluto. Al aplicar este esquema a la sociedad mexicana, Félix-Brasdefer (2009) destaca dos componentes centrales de la imagen sociocultural: uno de afiliación, la confianza, y otro de independencia, el respeto, nociones que ya se habían probado explicativas en estudios previos (Curcó 2014). El respeto predomina para "mostrar formalidad y consideración al otro" y se manifiesta en la reserva y la comunicación indirecta, sobre todo en situaciones en las que hay distancia social y una desigualdad en la relación de poder; la confianza, por otra parte, "se manifiesta a través de la franqueza mediante la cual los interlocutores expresan sus intenciones sin reserva y con un estilo directo de comunicación". Los estudios apuntan a que los mexicanos, en general, se orientan en clave de respeto y confianza hacia la preservación de la imagen positiva del hablante y de su interlocutor, "entendida efectivamente como imagen pública individual y no en términos de imagen grupal" (Curcó 2014:23). En términos de conducta verbal, se hace prevalecer el involucramiento sobre la independencia (Félix-Brasdefer 2006: 31). Esa "imagen pública individual" que incorpora tanto al hablante como al oyente puede entenderse también como un "yo relacional", en el que la salvaguarda de la imagen del otro tiene un puesto privilegiado: "un yo social, interactivo, según el cual se da prioridad a la otra persona y luego al hablante" (Félix-Brasdefer 2009: 15-16).

En esta dinámica, los cumplidos en el habla de Monterrey funcionan como actos valorizantes que realzan la imagen del otro y, al poner en evidencia la amabilidad del hablante, tienen también una repercusión positiva en la imagen propia. Puesto que el principal objetivo social del cumplido es "crear o mantener un ambiente de amabilidad" Haverkate (1994:82), vemos entonces que este acto de habla sirve para vehicular de forma directa la imagen de afiliación que caracteriza a la cultura mexicana y su estudio podrá por tanto aportar conclusiones lingüísticas importantes.

\section{LOS HALAGOS DEL CORPUS: TIPOS Y FUNCIONES}

Hemos encontrado un total de 68 secuencias de halago en el corpus PRESEEA y 59 en el de AMERESCO. Aunque la diferencia numérica no es grande, hay que tener en cuenta que el 
índice en AMERESCO es comparativamente muy superior, si pensamos que la duración de las entrevistas de este corpus coloquial es de 30 minutos frente a los 90 minutos de las entrevistas de PRESEEA. Una primera observación es entonces resaltar la mayor frecuencia de cumplidos en contextos discursivos informales, en los que el nivel de conexión y confianza entre los interlocutores es superior. Se verifica también la necesidad de incorporar corpus de habla espontánea en el estudio del halago, algo que sin embargo no ha caracterizado los estudios sobre el halago en México publicados hasta el momento.

En la clasificación de los halagos, hemos seguido una perspectiva de análisis conversacional y de su función en el discurso, de acuerdo con el esquema propuesto en Rodríguez y Jiménez (2010: 317) en su estudio del habla de Monterrey. Las funciones discursivas diferenciadas se presentan en un continuum de grados de cooperación en la co-construcción de la cortesía y comprenden: 1) halagos de colaboración conversacional; 2) halagos que vehiculan 0 sustituyen a otros actos de habla en el discurso; 3) halagos de afiliación o amistad. En la Figura 1 aparecen las incidencias de halagos en los dos corpus según la función discursiva:

Figura 1. Incidencias de halagos según la función discursiva

\begin{tabular}{|l|l|l|l|c|}
\hline & PRESEEA & AMERESCO & Casos & $\%$ \\
\hline Apoyo conversacional & 42 & 28 & 70 & 55.1 \\
\hline Macro-acto de discurso & 12 & 9 & 21 & 16.5 \\
\hline Amistad y afiliación & 14 & 22 & 36 & 28.3 \\
\hline TOTAL & 68 & 59 & 127 & \\
\hline
\end{tabular}

\subsection{GRUPO 1. HALAGOS DE APOYO A LA CONVERSACIÓN}

La función de estos cumplidos es mostrar interés y atención a lo dicho por el interlocutor para que éste mantenga su narración y no decaiga la comunicación. Aunque se registran en todas las lenguas, su frecuencia varía notablemente según la cultura. En el corpus de Monterrey, los marcadores de discurso de apoyo conversacional son constantes en casi todas las entrevistas. Hay innumerables ejemplos que van desde puras marcas de apoyo que no exigen respuesta y solo muestran el interés: $\mathrm{mmm}$, sí, ;Ah! /Aja/ Mjm/iAh! Sí, hasta la presencia de halagos propiamente dichos. Estos halagos contribuyen a la elaboración y desarrollo del tema y siguen así el principio de cooperación con el interlocutor en el contexto de la co-construcción del diálogo conversacional. Mientras que los marcadores discursivos simples como sí, $\mathrm{mmm}$ son signos que señalan interés y dejan constancia de que el oyente sigue el hilo de la conversación, los halagos añaden un sentido de aprobación o acuerdo con lo dicho por el interlocutor y no constituyen solo una muestra de apoyo. Este es el tipo más frecuentes en los dos corpus (61.7\% PRESEEA; 47.4\% AMERESCO), aunque en proporción superior en el caso de PRESEEA. En (1) un hombre joven cuenta que estuvo detenido un día y medio en la cárcel y el entrevistador, otro hombre joven, lo apoya verbalmente con marcas como "simón/sí/mjm". Su estancia en la cárcel resulta un tema delicado y por ello, seguramente, no recibe un apoyo más explícito por parte del entrevistador. En la conversación, este tipo de reacción por parte 
del interlocutor es lo que el hablante espera, y por ello proporciona toda una justificación. Solo cuando al final cuenta que al salir de la cárcel fue con su mamá a comer a un restaurante conocido, el entrevistador le da un cumplido (ah qué chido), como señal de aprobación. Este cumplido final sirve para preservar la imagen positiva del interlocutor que el propio contenido del texto ponía en tela de juicio. El elogio en este caso sirve para "reconstruir" la imagen positiva del interlocutor.

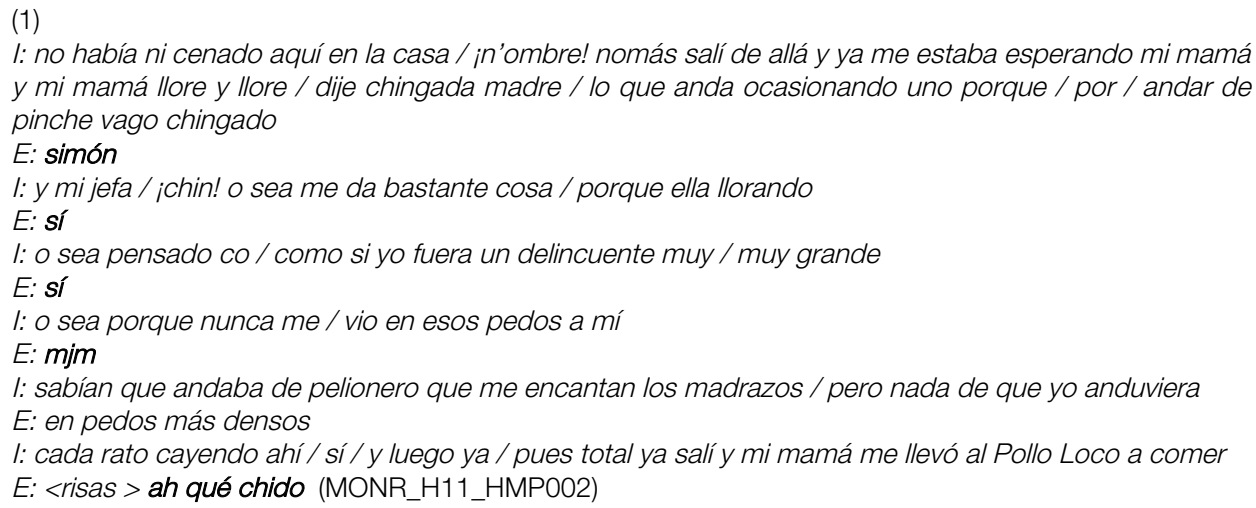

(2) proporciona otro ejemplo, en este caso en una entrevista coloquial, donde se aprecia el apoyo al programa en el que participan; en este caso, se va del simple apoyo al halago y al involucramiento personal.

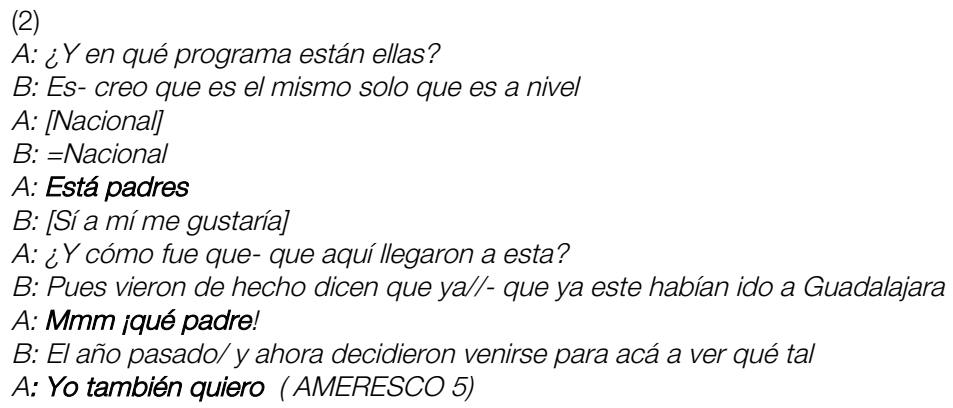

La diferencia en el porcentaje que supone este grupo en cada corpus se relaciona directamente con la diferente situación comunicativa. Los halagos de apoyo a la conversación representan la gran mayoría (61.7\%) del total de casos en PRESEEA. Es natural que el formato de entrevista semiformal de PRESEEA favorezca un elevado índice de halagos de este tipo, pues se trata de una situación en la que el/la entrevistador/a tiene un papel comunicativo condicionado y predeterminado y parte de este rol es garantizar que la conversación fluya con facilidad. En tal situación comunicativa, los apoyos conversacionales con seguridad aparecerán en un porcentaje superior al que se puede predecir para conversaciones de habla natural. Los marcadores de apoyo conversacional aparecen como muestras de cooperación a lo dicho por el otro, como cortesía, mientras que el halago se introduce cuando el interlocutor 
no solo escucha y apoya, sino que muestra su acuerdo ante lo expresado por el hablante, acto que introduce un significado diferente en el contexto conversacional. Ambos casos son claras manifestaciones de la imagen de afiliación, pues lo que impera es el mostrar interés por lo que dice la otra persona y el apoyo al punto de vista del interlocutor. En las entrevistas de PRESEEA estas son estrategias que el entrevistador tiene a su disposición para expresar la conexión y confianza con el interlocutor, si bien de una forma respetuosa.

\subsection{GRUPO 2: HALAGOS COMO NÚCLEO DE UN MACRO-ACTO DISCURSIVO}

Partiendo de la concepción de Van Dijk (1991) del macro-acto de discurso, Rodríguez y Jiménez (2014) recogen casos en los que el halago vehicula en el intercambio comunicativo una acción global distinta a la de "halagar", de forma que el halago aparece realizando la función de otros actos de habla, como por ejemplo el saludo, la felicitación, el agradecimiento, etc. Siguiendo un esquema similar, en nuestro corpus encontramos 21 casos en los que el halago aparece como núcleo de un macro-acto discursivo diferente, por ejemplo en (3) encontramos un acto de saludo muy utilizado en México (¡Qué bueno que __.!) y en (4) el halago se introduce con un significado de felicitación.

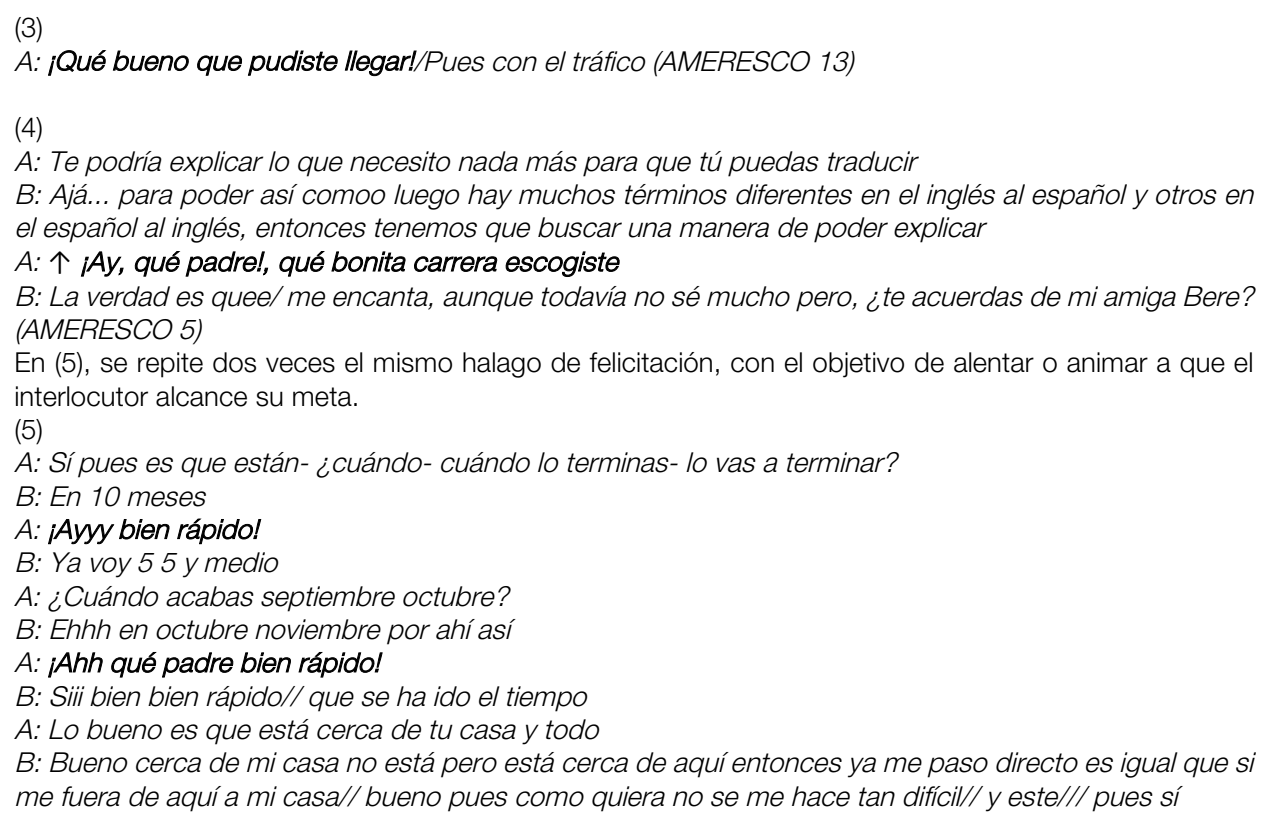

La proporción que este tipo supone en cada corpus es muy similar: 17.6\% (PRESEEA) y 15.2\% (AMERESCO). En su estudio sobre los cumplidos en el cine mexicano, Reyes (2014) recoge 21 casos (12.5\%) en los que el cumplido realiza el macro-acto de saludo. A medida que se realicen nuevos estudios de habla natural, podremos saber hasta qué punto el halago aparece como opción regular para determinados macro-actos de habla, como por ejemplo el saludo, 
en perjuicio de otras formas. Sería importante realizar también estudios comparativos que nos permitan comprobar si el uso del halago ejerciendo determinadas funciones podría considerarse como un uso característico de la cultura mexicana. En este grupo entra en juego la percepción positiva que se tiene del halago en esta comunidad, y que siempre se orienta a la preservación de la imagen de ambos interlocutores.

\subsection{GRUPO 3: HALAGOS DE AMISTAD Y AFILIACIÓN}

En este último grupo se incluyen aquellos halagos que contribuyen a la consolidación de lazos y valores comunes entre los interlocutores y que actúan como refuerzo de los lazos de afiliación al grupo o de amistad mutua. De esta manera, el hablante manifiesta su identidad en forma de afiliación a un grupo o a una relación cercana. Rodríguez y Jiménez (2014) encontraron que este tipo de halago es el más frecuente entre individuos de un mismo grupo social. El porcentaje que suponen en el total es superior en AMERESCO (37.2\%) que en PRESEEA (20.5\%); vemos que las conversaciones coloquiales entre personas que se conocen favorecen la presencia de estos halagos de filiación. En (6), (7) y (8) aparecen estos halagos cuando los hablantes hablan de sus compatriotas, algo que en general va acompañado de opiniones positivas. En todos estos ejemplos, se ensalza el elemento grupal de los mexicanos y se habla en sentido positivo de sus virtudes y valores. En (6) el elemento grupal incluye rasgos que caracterizan a todos los mexicanos y lo identifican con su patria, mientras que en (7) los halagos hacen referencia al valor común que es el amor a la familia.

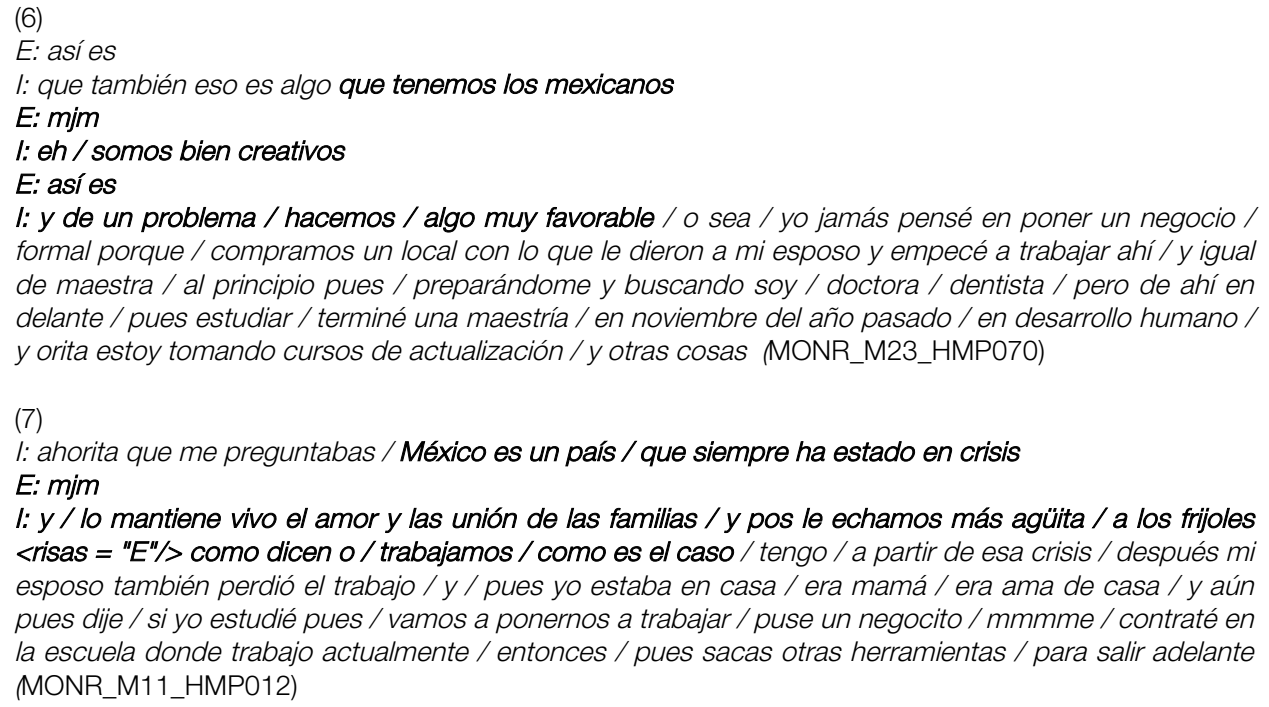
pues dije / si yo estudié pues / vamos a ponernos a trabajar / puse un negocito / $\mathrm{mmmme} \mathrm{/} \mathrm{contraté} \mathrm{en}$ la escuela donde trabajo actualmente / entonces / pues sacas otras herramientas / para salir adelante MONR_M11_HMP012)

En (8) la hablante ensalza sus habilidades de buena madre pero afirmando después que cualquiera puede hacerlo; lleva a cabo la generalización a través del uso de "tú" inclusivo de grupo.

(8)

l: y / y te lo / te lo digo no / con el afán de presumirte / sino con el afán de que tú misma te des la respuesta / ¿sí? / tengo / dos carros / el de mi hija / y el mío / ¿sí? / y tengo / este / dos hijas / en escuelas 
particulares / ¿sí? / y eso está pagando la universidad / entonces / yo no sé / si será / mucho / o si sea poco lo que gano / a lo mejor sí he sabido mucho / administrar / y más que todo / que como saber a qué / a qué le das importancia

E: Pues es usted una buena madre

I: ay gracias / nomás es de que quieras MONR_M13_HMP033)

Un ejemplo de AMERESCO (9) muestra la filiación con halago y luego ensalza a su interlocutor como

"una de las pocas personas" que tiene la cualidad de comprender, como un ser "único".

(9)

A: Claro, porque no puedes traducir unaa 个 jimagínate!, que tienes que traducir a lo mejor un guión para una caricatura para niños y un guión para una caricatura de adultos, entonces no vas a llamar a las cosas de una misma manera porque los niños no van a entender a lo mejor todo y los adultos a lo mejor se les va a ser muy tonto si lo dejan con ese lenguaje, tienen que hacer algo para ellos, jahl, pero, iqué padre! $B: \uparrow$ eres de las pocas personas que realmente entienden eso, o sea, es que muchas personas, te lo juro, que piensan que es asi como que... "Ay, por favor, es súper fácil", pero no se dan cuenta que realmente como que, hay reglas, o sea, hay un por qué de las cosa es comoo/ muchas personas critican sobre todo, el momento dee la traducción del nombre de las películas pero, o sea, porqué no las dejan como estaban en inglés y bueno... ¿sabes por qué hacen eso? (AMERESCO 2)

En la tipología de los halagos propios del habla regiomontana, hemos visto que en el corpus de PRESEEA la incidencia de halagos de apoyo conversacional es muy alta, en relación a los otros dos tipos, lo que nos indica que en entrevistas semiformales, prevalecen los apoyos conversacionales, más respetuosos, frente a los relativos a otros actos de habla y a los de filiación, por corresponder estos últimos a un ámbito más familiar y de confianza. Es por ello que en las entrevistas del corpus coloquial de AMERESCO el tipo 1 y 3 son los más numerosos, sin existir grandes diferencias entre ellos. El análisis de este último corpus, mucho más coloquial y espontáneo, aporta importantes datos a la categorización y contextualización del halago en México.

\section{ESTRATEGIAS DE CORTESÍA EN LA RESPUESTA AL HALAGO}

Como todo acto de habla interactivo, el cumplido presenta dos caras diferenciadas: en la primera parte aparece un enunciado con el elogio y, en la segunda, otro enunciado (o enunciados) que responda adecuadamente a la alabanza. El cumplido y la respuesta al cumplido pueden entenderse como dos actos de habla que juntos componen un único evento comunicativo y, por lo tanto, deben ser considerados juntamente (Briz, 2004). Para Flores (2014: 236) estos dos actos reflejan los valores culturales de una comunidad lingüística "porque muestran cómo los hablantes reaccionan a juicios asertivos sobre su identidad personal y social. Saber expresar un cumplido es tan importante como saber responderlo de manera adecuada". La respuesta, sin embargo, es a veces de difícil negociación. Pomerantz (1978) explica el dilema que afronta el hablante al responder a un cumplido: si está de acuerdo con el interlocutor y acepta el halago, el hablante cae en el auto-halago, y puede parecer arrogante y presuntuoso, pero si lo rechaza, contradice a su interlocutor, lo que amenaza el acercamiento y la solidaridad entre ambos. Tenemos que ver entonces cuál es el papel de las estrategias de cortesía para que la respuesta no dañe la imagen del otro y, al no quedar bien con el otro, se dañe también nuestra propia imagen. En el sistema de co-construcción del diálogo entre hablante e interlocutor, la atenuación del auto-halago se presenta como una muestra de cortesía en México. Mediante esta estrategia el hablante quita importancia a sus 
logros o propiedades y se desvaloriza, para, por otra parte, ensalzar al otro.

En (10) el hablante acepta el halago, pero lo mitiga al responder con otro halago. Este tipo de respuesta busca restablecer la igualdad entre los hablantes, que se vería comprometida con una directa aceptación del halago.

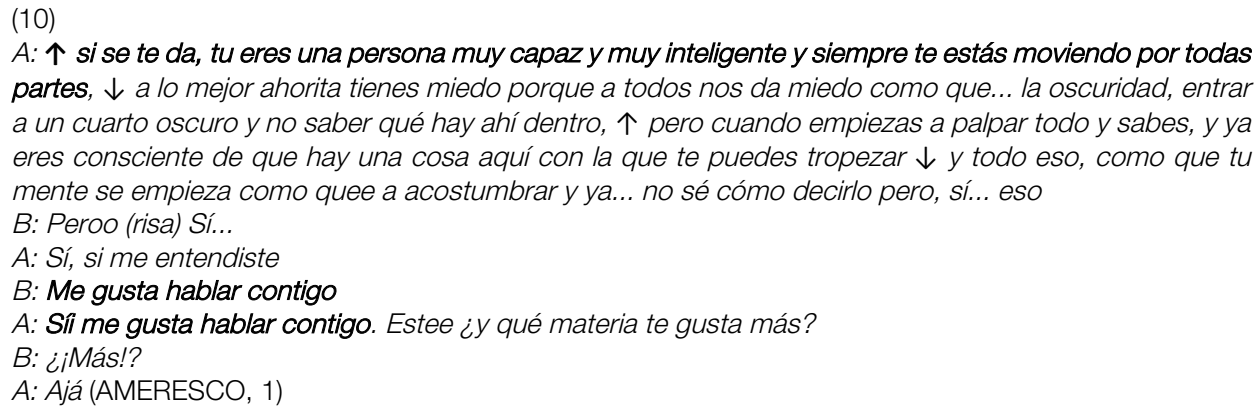

Observamos que en las entrevistas semi-formales existen más casos de secuencias de solo dos turnos, en las que el interlocutor agradece el halago y cierra el turno de palabra. Este fenómeno es menos frecuente en las entrevistas coloquiales, donde el acto de halagar tiene lugar en un número de turnos de tres ó más intervenciones, en los que a menudo se dan atenuaciones, justificaciones o elementos de humor que contribuyen a marcar la solidaridad entre los participantes. En estas secuencias se recurre con frecuencia a la repetición, tal vez con el fin de remarcar estos propósitos:

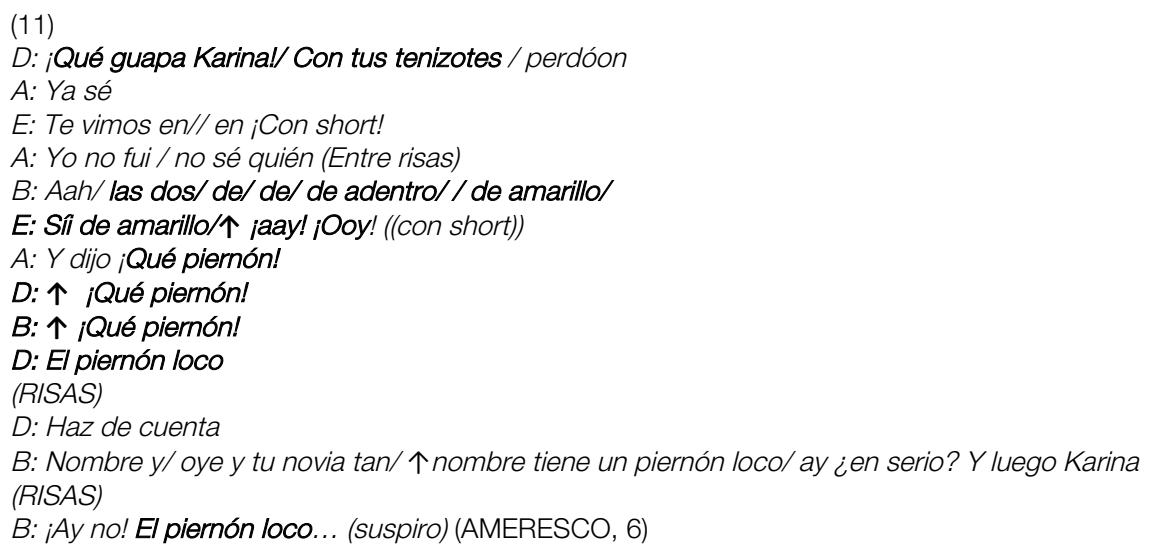

Como vemos, la aceptación del halago se realiza en muchos casos de forma mitigada (Yo no fui, no sé quién es), por lo que podríamos hablar de una cortesía mitigadora en la que se suaviza el halago minimizándolo, y siempre a través de estrategias en las que se incluya al otro en el grupo para igualarse a él. Esta atenuación del halago puede verse como marca de cortesía en el habla en los hablantes de Monterrey: aminoran cualidades, actitudes y acciones del "yo" en un intento de minimizar la alabanza recibida. Algunos recursos morfosintácticos para alcanzar este fin son el uso de diminutivos, la modalización no asumida que elude el "yo" 
y lo sustituye por otro sujeto impersonal o colectivo (se, uno, nosotros), o las largas justificaciones. Cuando el mérito es propio se tiende a impersonalizar (uno en 12) por apego al grupo y por el deseo constante de expresar la conformidad con su situación. En (12), el hablante disminuye el valor de su trabajo mediante el uso de diminutivos y explicando el hecho de que le va bien por el número de años que lleva allí y porque el trabajo es muy sencillo. El diminutivo en (13) pretende también restar importancia al logro personal; la entrevistada minimiza el mérito de haber pasado a la Secundaria, a pesar de que se deduce por el contexto que pocas personas lo consiguieron.

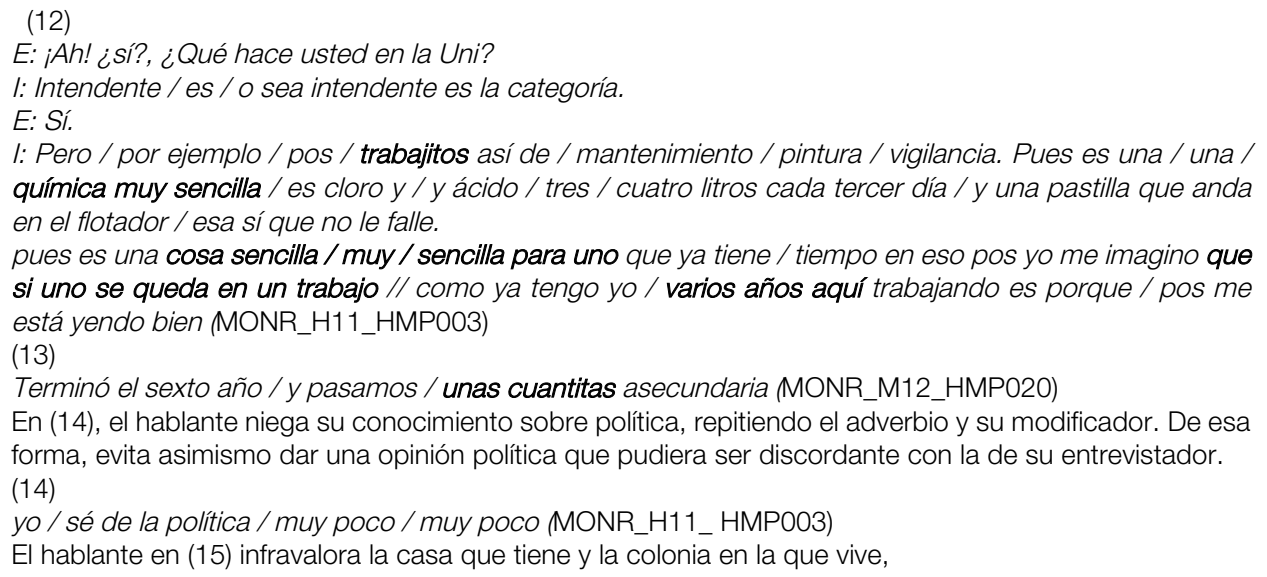

mencionando primero que es "media" y luego "media baja" y que pudo comprarla porque le daban crédito a todo el mundo. Se sitúa, incluso, por debajo de su nivel social real para no parecer pretencioso. En este caso, la estrategia de evitación del auto halago es dar una larga explicación que justifique la constitución de un logro que pudiera ser evaluado como individual y fuera del alcance del grupo al que pertenece el hablante. Se observa también, una vez más, que este proceso se actualiza lingüísticamente mediante la modalización no asumida que elude el "yo" por otro sujeto (cualquiera) y con largas justificaciones.

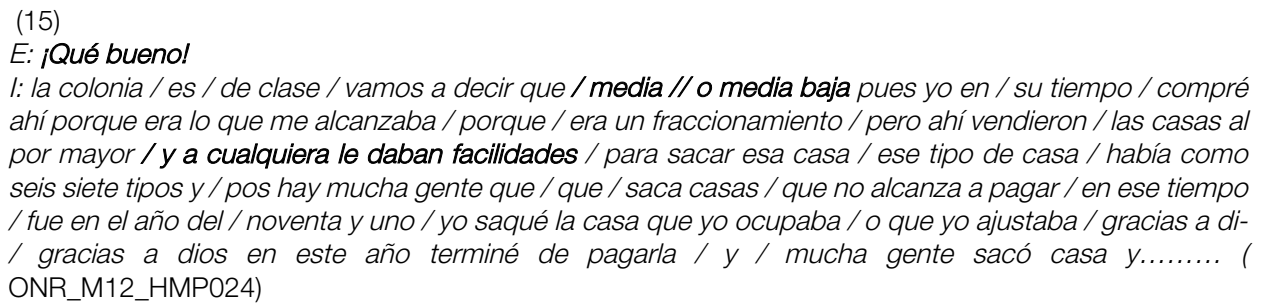
ahi porque era lo que me alcanzaba / porque / era un fraccionamiento / pero ahí vendieron / las casas al por mayor / y a cualquiera le daban facilidades / para sacar esa casa / ese tipo de casa / había como seis siete tipos y / pos hay mucha gente que / que / saca casas / que no alcanza a pagar / en ese tiempo / fue en el año del / noventa y uno / yo saqué la casa que yo ocupaba / o que yo ajustaba / gracias a di/ gracias a dios en este año terminé de pagarla / y / mucha gente sacó casa y........ ( ONR_M12_HMP024)

En (16), el hablante acepta el cumplido por cortesía, ampliándolo a su grupo familiar, para luego desviarlo identificándolo con un defecto, "ser bajitos", y llamando la atención sobre las negativas consecuencias que puede deparar el parecer joven. Todas estas estrategias se encaminan a evitar el auto-halago. 
l: estás muy joven / te ves más joven ¿eh? / todavía / ¿sí? / ¿te han dicho?

E: sí / sí me han dicho / de hecho todos en mi casa / nos vemos bien jovencitos / yo creo que como somos bajitos

l: sípero no tu / tu piel / así todo tu / tu expresión de / y / y / este la expresión de tus ojos / es todo como I muy sano todo muy de niña /

E: sí gracias / me da miedo porque a veces / digo no se me vayan a venir de repente todos los años de golpe / y de repente / no estoy preparada para verme tan grande

I: no / pero yo creo que no / yo creo (ONR_M13_HMP03)

A continuación, vemos cómo el hablante describe su casa como "pequeña" y explica que tiene "pocos" amigos. Esta manera de minimizar sus logros permite al hablante no caer en el autohalago en su respuesta.

(17)

E: ¿era de otro nivel / cuando se compró?

I: pues sí E: ¿era de otro nivel / cuando se compró?

l: pues si

l: pues mi casa / es pequeña(...)

l: bueno / amigos / la verdad que ya con el tiempo te das cuenta que amigos / que son contados // yo

he apostado más / este / en este caso por mi familia / yo puedo decir que mis amigos a lo mejor son mis

hermanos / este / que es con quien más convivo (MONR_H12_HMP016)

También encontramos casos en los que el halago se acepta sin estrategias mitigadoras, como en (18). Es interesante que en este caso parece producirse una tensión entre dos valores culturales que compiten; por una parte, opera la inadecuación social del alabarse a sí mismo "elemento base de la cultura de la modestia y sobriedad del norte de México" (Rodríguez y Jiménez, 2010: 143) pero, por otra, se trata del valor cultural que postula que toda madre debe expresar un sentimiento de orgullo por sus hijos. Este segundo valor vence en este caso y explica la aceptación del halago de la entrevistadora y el auto-halago que sigue:

(18)

E: iyl qué / qué quapos y qué bonita es su hija

I: Que te puedo decir yo que'stán hermosos mis hijos / preciosos / bien parecidos / n'hombrel Valen oro MONR_M11_HMP012)

(19) y (20) presentan otros casos de aceptación del halago. Otra vez, la aceptación va unida a la afirmación de valores culturales compartidos. En (19) el éxito se explica porque la hablante ha sabido administrar su capital para poder proporcionar una buena educación a sus hijos, ha sabido "a qué dar importancia" y los logros se han conseguido a través del esfuerzo. La educación de los hijos y la cultura del esfuerzo y trabajo personal son valores que la comunidad mexicana comparte y el participar de ellos es un salvoconducto que permite presentar el logro personal desde una perspectiva comunitaria.

(19)

l: y / y te lo / te lo digo no / con el afán de presumirte / sino con el afán de que tú misma te des la respuesta / ¿sí? / tengo / dos carros / el de mi hija / y el mí / ¿sí? / y tengo / este / dos hijas / en escuelas particulares / ¿sí? / y eso está pagando la universidad / entonces / yo no sé / si será / mucho / o si sea poco lo que gano / a lo mejor sí he sabido mucho / administrar / y más que todo / que como saber a qué / a qué le das importancia

Pues me ha ido bien/trabajando muy duro/no me quejo (MONR_H31_HMP076) 
En el siguiente caso de AMERESCO la hablante se autohalaga, pero en un contexto de broma, que diluye el halago en el grupo.

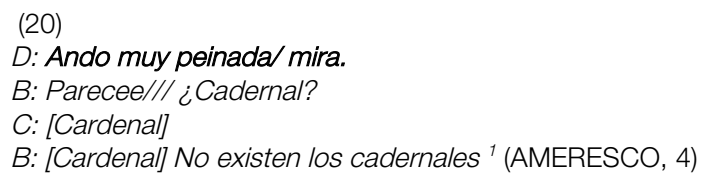

Finalmente, tenemos los casos de rechazo implícito o explícito del halago. Se trata del grupo más reducido, que supone el $5 \%$ del total. En (21) los interlocutores A y B halagan la habilidad de $\mathrm{C}$ para las manualidades y ésta elude los cumplidos explícitamente (Ay Alicia) o mediante el silencio $(\mathrm{mmmmm})$. Pareciera que no está de acuerdo con lo alabado, a pesar de la insistencia de las otras dos participantes.

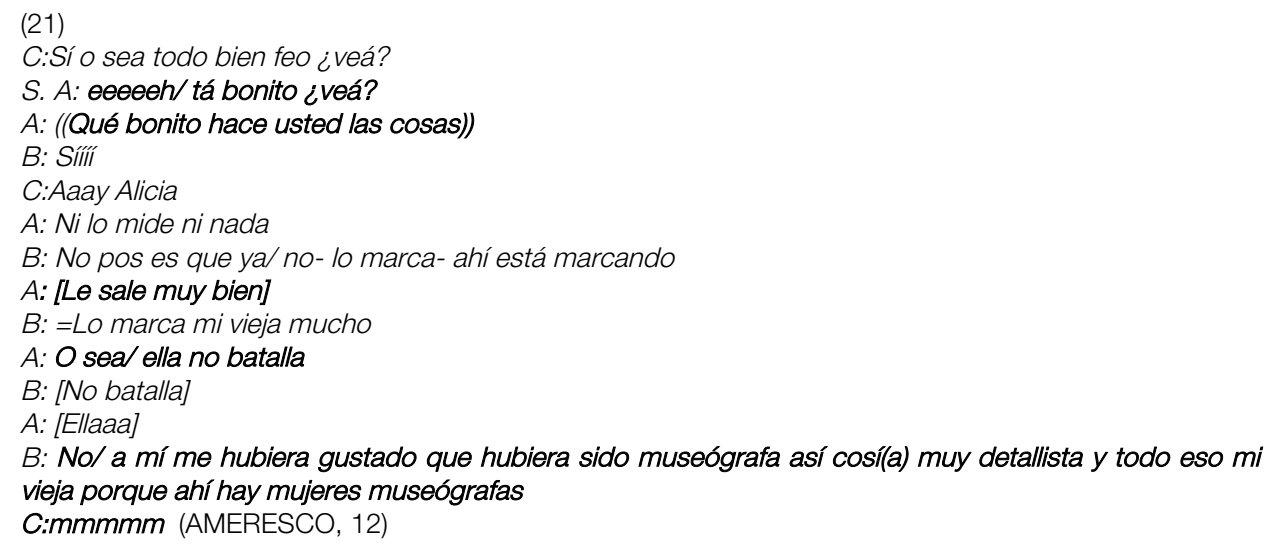

(22) presenta un ejemplo de rechazo explícito; Ya sea como objeto de broma o no, parece que la persona que recibe el halago no está de acuerdo con el objeto del mismo, por lo que se calla o lo rechaza.

\section{(22)}

E: Me acuerdo cuando/ este entré yo al taller allá en Sabinas///(3") ya me presentaron ahí las- las dueñas del taller y todo que dijo esta muchacha aquí viene a trabajar/ con nosotros///(3") se pararon y aplaudieron y todo/Y luego se quedó una de ellas// viendo

B: ;Chelo para diputada! / /Chelo para diputada! $\uparrow$

C: i((Cállate))/ hombrel «entre risas» (AMERESCO, 5)

A continuación, veremos nuestros resultados a la luz de otros dos trabajos dedicados a la respuesta al cumplido en el español de México (Flores 2014; Reyes 2014). Los resultados de Flores (2014) sobre las respuestas al cumplido en Puebla revelan que la aceptación del cumplido (46\%) es más frecuente que su mitigación (34\%) o rechazo (20\%). Dentro de la aceptación, el agradecimiento se recoge como la sub-estrategia más común (15\%). El agradecimiento se recoge incluso en casos de rechazo o desviaciones, lo que pone de

\footnotetext{
${ }^{1}$ Está imitando/arremedando a alguien.
} 
manifiesto que mostrar gratitud es un elemento necesario del comportamiento cortés en la cultura mexicana, y su ausencia sería interpretada como una marca de descortesía. Como vimos, nuestros resultados apuntaban en la misma dirección. La mitigación fue la segunda estrategia más usada en el estudio (34\%) y, otra vez como en nuestros ejemplos, se trata de evitar el auto-halago y para ello se dan explicaciones que impersonalizan o minimizan las virtudes objeto de halago. Finalmente, en el $20 \%$ de los casos, el halagado teme que aceptar el halago comprometa su situación en el grupo por parecer arrogante y, para ser aceptado adopta un comportamiento modesto y lo rechaza. De esta forma, "rechazar un cumplido se puede considerar como un acto anticortés donde el objetivo principal es buscar la aceptación del grupo" (Reyes, 2014: 236). Así vemos cómo agradecer el cumplido, mitigarlo y rechazarlo son formas que el mexicano usa para salvaguardar la imagen del otro y al mismo tiempo su propia imagen. Flores (2014) propone que el grado de intimidad entre los participantes funciona como una variable que influye en el tipo de estrategia que se usa para responder a un cumplido. Por ejemplo, "Ios hablantes agradecen más cuando el otro interlocutor es un conocido (...) cuya relación no es muy cercana con el interlocutor" (Flores 2014: 259). Este factor se constata como relevante en nuestro corpus y la mayor distancia social entre los hablantes explicaba el más alto índice de agradecimiento en el corpus de entrevistas semiformales. En el corpus de PRESEEA la respuesta más frecuente al halago es el agradecimiento (58\%). Esta respuesta, sin embargo, solo se da en AMERESCO el 29\% de los casos. La diferencia en el grado de intimidad entre los hablantes parece ser aquí un factor determinante. El tema objeto de elogio es otra variable que afecta al tipo de respuesta que recibe el halago. Cuando el cumplido elogia la apariencia física y se da en una relación cercana, es muy frecuente la mitigación y el rechazo, que sirven para salvaguardar la imagen positiva del otro. En nuestro corpus, el elogio de una cualidad física recibía también una respuesta mitigada (16) aunque en este caso se trataba de la relación semiformal entre entrevistador/entrevistado.

Un segundo estudio es el que lleva a cabo Reyes (2014) sobre los cumplidos presentes en 22 películas mexicanas rodadas en la década del año 2000 al 2010. Reyes reporta que en el 44.8 $\%$ de los casos los mexicanos buscan la afiliación con el oyente en su respuesta a los cumplidos, con respuestas que aceptan, agradecen, buscan confirmación, exaltan y devuelven el cumplido. Por otra parte, en un total del 27.36\% de las respuestas, los hablantes dan explicaciones, minimizan el cumplido, lo rechazan, lo contradicen y lo corrigen; en estos casos lo que prevalece no es la necesidad de afiliación con el oyente sino el deseo de evitar el autohalago.

En conclusión, hemos visto que en general, en ambos corpus, el halago se acepta, pero de forma mitigada. En los casos en que se acepta de forma directa, lo que se admira es un valor social colectivo mexicano, por ejemplo, el orgullo materno por los hijos o los logros a través del esfuerzo, de forma que lo positivo pasa de ser individual a ser colectivo. Tanto nuestros datos como otros estudios confirman que el agradecimiento, la mitigación y el rechazo del cumplido aparecen como estrategias para reafirmar la imagen propia, pero de forma que a la vez se promueve la imagen del otro o los valores del grupo. 


\section{LA ESTRUCTURA SINTÁCTICA DEL HALAGO}

En su tesis doctoral sobre la producción del halago en estudiantes americanos de español como segunda lengua, Hasler-Baker (2013: 34-38) hace un repaso de la estructura sintáctica de los cumplidos recogidos en una serie de estudios sobre el halago en español. Llama la atención que en todos los corpus la gran mayoría de los halagos repiten un número muy reducido de estructuras sintácticas. Si se comparan los datos de todos estos corpus, resulta que las estructuras sintácticas más comunes en el español de Puerto Rico, Costa Rica, México y Ecuador son relativamente similares (37). El español de los cuatro países presenta una versión de Qué + Adjetivo como la construcción más frecuente y en muchas aparece el uso de una frase del tipo Me gusta/encanta + Adjetivo o Te/Se queda + Adjetivo. El resto de las fórmulas tienen una estructura bastante similar, y constan de un SN + V (sobre todo ser, estar, o parecer + + Adjetivo. En la mayoría de estas fórmulas, el adjetivo o el adverbio incluido es el encargado de transmitir la carga semántica. En el español de México y Costa Rica, la mayor parte de los halagos adjetivales o adverbiales contienen bien o bonito; en Ecuador, los adjetivos más comunes fueron bien, bonito, y lindo y en Puerto Rico bien, bonito, lindo y excelente (38).

En su estudio del halago en el habla de Monterrey, Rodríguez y Jiménez (2010: 183) hallan huella de las estructuras ritualizadas descritas por Hasler-Baker, entre ellas Qué + Adjetivo (“¡Qué guapa!”, "¡Qué bonito nombre!”, "¡Qué padre que fue una de las fundadoras de aquí!”), $V P+N P$ [Adj (Nombre)] (iTienes una hija muy linda!), Te/Se queda + Adjetivo (jTe queda bien rico!) y (Pro)+VP+(Intensificador) Adjetivo/Adverbio (¡Ustedes tocan muy bien!). Este tipo de estructuras daban cuenta del $74 \%$ de los cumplidos recopilados en su corpus de entrevistas de PRESEEA-Monterrey.

De la misma forma, en el presente estudio identificamos que la gran mayoría de los halagos del corpus repiten fórmulas similares. Ahora bien, se observa que la presencia de estas estructuras es superior en el caso de PRESEEA (83\%) que en las conversaciones coloquiales de AMERESCO (62\%). Esta diferencia sugiere que en contextos informales y entre interlocutores cercanos, los hablantes expresan el elogio con una mayor creatividad e individualidad. Veamos a continuación algunos ejemplos de fórmulas de halago halladas en ambos corpus.

\section{En PRESEEA}

Qué +adj: ¡Qué padre!

Te/Se queda + Adjetivo:jTe queda muy bien!

(Pro)+VP+(Intensificador) Adjetivo: Es muy estudiosa

\section{En AMERESCO:}

Me gusta+SN : Me gusta hablar contigo

(Nombre) SV [Verbo+SN]:Sí, se te da, eres una persona muy capaz

Eres una de las pocas personas que entiende esto

¡Tan+adjetivo!: Mi carnal, tan galán se ve, ¿Sí o no?

Tan hermosa que eres y ahí con esas cosas feas

Normas (ISSN: 2174-7245) |

https://ojs.uv.es/index.php/normas/index

Junio 2017 | Volumen 7 | Número 1| Pág.183 
SN [ser/estar/parecer]+(Intesinficador)+Adjetivo: Está muy guapo también Caril

Pues es muy bonito eso, mi amor

Yo soy muy platicadora

En el corpus de conversaciones espontáneas se recogen estructuras que conllevan un significado de "alabanza" que es necesario inferir a partir del contexto conversacional concreto, pues los enunciados en sí, a diferencia de las estructuras de halago más fosilizadas, no confieren en sí mismos esa carga semántica. Estas formas se componen sobre todo de enunciados declarativos que se interpretan como halagos a la luz de su función en el contexto de la conversación. A continuación, incluimos algunos ejemplos, cuyos contextos se encuentran en fragmentos citados previamente.

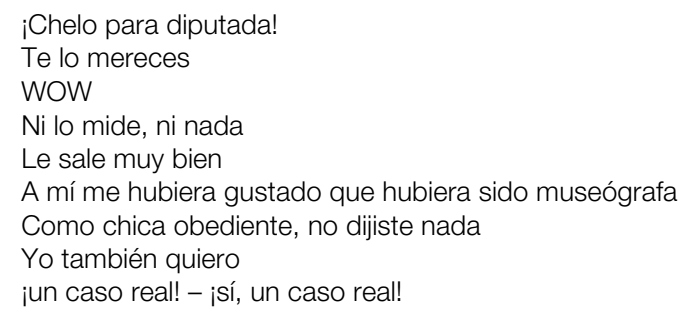

Es preciso, pues, analizar los enunciados a nivel de análisis conversacional para determinar su función como acto de habla en el discurso, más allá de su estructura sintáctica. En este sentido, en la muestra del corpus coloquial aparece una mayor variedad de formas de expresar los cumplidos, más creativas y originales, que se alejan de las fórmulas convencionales; estos cumplidos aparecen en fases más largas, con repeticiones, elementos de humor y un número mayor de intervenciones conducentes a la filiación.

\section{CONCLUSIONES}

A partir de éste y anteriores estudios podría decirse que el sistema de cortesía mexicano está orientado principalmente a reforzar la imagen de filiación por la que el hablante pretende ser valorado y aceptado por los demás miembros del grupo, por lo cual protege su imagen y la del otro, en un deseo de "quedar bien", a través del apoyo a las opiniones y actos del otro, mostrando su interés en él y halagando como modo de mostrar sentimientos de solidaridad y amistad. La cultura mexicana se ha caracterizado como una cultura de acercamiento, porque promueve actos valorizantes como halagos, cumplidos, piropos, de modo que siguen el ideoma "hispánico" de que "hay que halagar" (Briz 2004: 82-83).

En el presente artículo hemos profundizado en el estudio del halago en la comunidad de Monterrey, añadiendo un corpus de entrevistas espontáneas en las cuales aparecen halagos coloquiales en el contexto de una conversación informal. Como se ha visto, el grado de formalidad de la situación comunicativa tiene un claro impacto en la aparición de halagos en el discurso: nuestra investigación muestra una mayor frecuencia de cumplidos en conversaciones espontáneas de discurso no planificado (con repeticiones, interrupciones, 
lenguaje informal, etc.), en las que el nivel de conexión y confianza entre los interlocutores es superior. El grado de formalidad no sólo aparece afectando la frecuencia de halagos, sino también el tipo de función discursiva que desempeñan y su misma estructura sintáctica. Mientras que los halagos de apoyo a la conversación representaban la gran mayoría (61.7\%) del total de casos en PRESEEA, en las entrevistas del corpus coloquial de AMERESCO los halagos de apoyo a la conversación y los de amistad y afiliación presentan una frecuencia similar. Es evidente que en el formato de entrevista semiformal de PRESEEA, el rol del entrevistador es en parte garantizar que la conversación fluya con facilidad, lo que propicia sobremanera la aparición de ese tipo de halago. Desde el punto de vista de su estructura sintáctica, la aparición de fórmulas más o menos ritualizadas es muy superior, como es de esperar, en las estrevistas semiformales que en las más coloquiales.

A partir de la interpretación de nuestros resultados corroboramos, con Arundale (1999: 119153), que el halago en Monterrey se realiza dentro de un proceso de co-construcción en el que tiene lugar una negociación de la imagen, que protege tanto la imagen propia como la del otro. De esta prioridad se parte para explicar la estructura, tipos y respuestas al halago, como acto de habla muy relevante en el contexto de esta cultura. Sugerimos que el halago es una intervención que se prefiere sobre otras por constituir siempre una muestra de cortesía valorizante de significado positivo para el interlocutor, ya sea como apoyo a la conversación, como muestra de afiliación o en sustitución de otros actos. Por ello, ese intercambio interactivo resulta tan complejo, pues se va co-construyendo en la conversación para que exista siempre un equilibrio entre ambos participantes y no se dé una imposición de uno sobre el otro. En una sociedad en la que predomina el estilo de habla indirecto, se podría proponer que el halago se emite en una secuencia en la que: 1) se trata de mostrar aprobación de una acción, conducta u opinión; 2) en el caso de apoyar una conversación o contribuir a la elaboración del tema conversacional; 3) o puede constituir una marca de reforzamiento de halago cuando se produce la repetición del mismo, como un modo de corroborar lo afirmado como algo fidedigno y no solo como una forma de cortesía.

Si comparamos los dos corpus de nuestra investigación, vemos que en el corpus AMERESCO hay muchas apariciones de halagos con fines afiliativos para mostrar solidaridad grupal y se aprecian asimismo, varios turnos en las secuencias de halagos, en número mayor al observado en similares actos de habla en otras culturas. La respuesta al halago en esta comunidad es generalmente de aceptación, con la marca de agradecimiento en contextos más formales y con una aceptación implícita o explícita que generalmente atenúa el halago, minimizándolo o dando una larga justificación que excluye su valor a favor del grupo que lo apoya. El agradecimiento, la mitigación y el rechazo del cumplido aparecen como estrategias para reafirmar la imagen propia, pero de forma que a la vez se promueva la imagen del otro o los valores del grupo.

En conclusión, se ha estudiado la concepción que en cada contexto tiene el hablante del cuidado de su imagen y de la imagen de los otros en relación con la cortesía. A partir de los datos, se han hecho propuestas sobre el acto de halagar y su realización en intercambios 
comunicativos que varían según cambian los interlocutores. Es importante que estudios futuros identifiquen la estructura concreta de cada situación de enunciación de esta forma de cortesía valorizante, sus diversos usos, fines, destinatarios y/o el grupo social protagonista, de manera que podamos comparar su uso en esta comunidad de Monterrey con el de otras comunidades en el contexto mexicano y en otras culturas hispanohablantes.

\section{BIBLIOGRAFIA:}

ARUNDALE, Robert (1999): «An alternative model and ideology in communication for an alternative to politeness theory", Pragmatics 9:1, 119-153.

BERNAL, María (2007): Categorización sociopragmática de la cortesía y de la descortesía. Un estudio de la conversación coloquial española, Tesis doctoral, Estocolmo, Universidad de Estocolmo.

Bravo, Diana (1999): « ¿lmagen positiva vs. imagen negativa?, Pragmática sociocultural $y$ componente de face», Oralia: Análisis del discurso oral 2, 155-184.

Bravo, Diana (2005): Estudios de la (des)cortesia en español: categorias conceptuales y sus aplicaciones a corpus orales y escrito, Buenos Aires, Programa EDICE.

BrAvo, Diana (2010): «Pragmática socio-cultural: La configuración de la imagen social como premisa socio-cultural para la interpretación de actividades verbales y no verbales de imagen», en F. Orletti y L. Mariottini (eds), (Des)cortesía en español. Espacios teóricos y metodológicos para su estudio, Roma-Estocolmo, Università degli Studi Roma Tre-EDICE, 19-46.

BRIZ GÓMEZ, Antonio (2004): "Cortesía verbal codificada y cortesía verbal interpretada en la conversación», en D. Bravo y A. Briz (eds), Pragmática Sociocultural: Estudios sobre el discurso de cortesía en español, Barcelona, Ariel, 67-92.

BRIZ GÓMEZ, Antonio (2006): «Atenuación y cortesía en la conversación coloquial: su tratamiento en la case de ELE", Actas del programa de formación para profesorado de ELE 2005-2006. Madrid, Instituto Cervantes, 9-49.

Brown, Penélope y Stephen LEVINSON (1987): Politeness. Some Universals in Language Usage, Cambridge University Press.

Bustos, Juan Antonio (2007): «Cumplidos», CIRCULO de Lingüística Aplicada a la Comunicación 31, 3-12.

CuRcó, Carmen (2007): «Positive face, Group Face and Affiliation: An overview of politeness studies on Mexican Spanish», en M.E. Placencia y C. García (eds.), Research on Politeness in the Spanish-Speaking World, New Jersey, Lawrence Erlbaum Associates, 91-120.

CuRCó, Carmen (2014): «Un comentario en torno a la noción de imagen", en J.M. Infante y M.E. Flores (eds), La (des)cortesía en el discurso, MonterreyEstocolmo: UANL-EDICE, 19-52.

FANT, Lars (1989): Cultural mismatch in conversation: Spanish and Scandinavian communicative behaviour in negotiation settings, Hermes.

FÉLIX-BRASDEFER, César (2006): « Linguistic politeness in Mexico: Refusal strategies among male speakers of Mexican Spanish", Journal of Pragmatics 38(12), 2158-2187.

FÉLIX-BRAsdefER, César (2008): Politeness in Mexico and the United States, Amsterdam/Philadelphia, John Benjamins Publishing Company.

FÉLIX-BRASDEFER, César (2009): «Estado de la cuestión sobre el discurso de la (des)cortesía y la imagen social en México. Perspectivas teóricas y metodológicas», en L Rodriguez Alfano (ed.), La descortesía y la imagen social en México, Estudios semiótico-discursivos desde varios enfoques analiticos, Nuevo León, México: FFyL, Universidad Autónoma de Nuevo León-Programa EDICE, 15-45.

FLORES, Elizabeth (2014): «Respuestas a cumplidos en el español de puebla», en J.M. Infante y M.E. Flores (eds), La (des)cortesía en el discurso, Monterrey-Estocolmo: UANL-EDICE, 235-266.

GOFFFMAN, Erving (1967): Interaction ritual: Essays on face-to-face behavior, New York, Doubleday Anchor.

HAVERKATE, Henk (1994): La cortesía verbal: Estudio pragmalingüístico, Madrid, Gredos.

HASLER-BARKER, Maria (2013): Effects of Pedagogical Intervention on the Production of the Compliment-Compliment Response Sequence by Second Language Learners of Spanish, Indiana University, PhD Dissertation.

HERNÁNDEZ FLORES, Nieves (2004): «La cortesía como la búsqueda del equilibrio de la imagen social», en 
D. Bravo y A. Briz (eds), Pragmática Sociocultural: Estudios sobre el discurso de cortesía en español, Barcelona, Ariel, 95-108.

LEECH, Geoffrey (1983): Principles of pragmatics, New York, Longman.

POMERANTZ, Anne (1978): "Compliment responses: Notes on the cooperation of multiple constraints", en Jim Schenkein (ed).Studies in the organization of conversational interaction, New York, Academic Press, 79-112.

REYES, María (2014): «QQué gusto verte! El acto del cumplido, su respuesta y su comportamiento cortés en diálogos cinematográficos mexicanos", en J.M. Infante y M.E. Flores (eds), La (des)cortesía en el discurso, MonterreyEstocolmo: UANL-EDICE, 181-234.

RODRíGUEZ, Lidia y Elena JIMÉNEZ (2010): «El halago en boca propia es vituperio. Usos del halago y de "ándale" como estrategias de cortesía en el Habla de Monterrey"», en F. Orletti y L. Mariottini (eds), (Des)cortesía en español. Espacios teóricos y metodológicos para su estudio, RomaEstocolmo, Università degli Studi Roma TreEDICE, 131-150.

RODRÍGUEZ, Lidia y Elena JIMÉNEZ (2014): « La variable social en el halago. Aplicación de un test de hábitos sociales en la comunidad de monterrey", en J.M. Infante y M.E. Flores (eds), La (des)cortesía en el discurso, MonterreyEstocolmo: UANL-EDICE, 297-322.

SCOLlon, Ron y Suzanne SCOLLON (1995): Intercultural communication, Oxford, Blackwell.

VAN DIJK, Teun (1991): Estructuras y funciones del discurso. México. 